\title{
Análise da Assistência Farmacêutica Hospitalar na linha de frente no combate a COVID-19
}

\section{Analysis of Hospital Pharmaceutical Care on the front line in the fight against COVID-19}

Pamela Cristina Coelho dos Reis ${ }^{1}$, Laís Barreira da Silva², Jéssyka Viana Valadares Franco ${ }^{3}$, Halline Cardoso Jurema ${ }^{4}$

\section{RESUMO}

Os farmacêuticos hospitalares tem papel fundamental em períodos de crise epidêmica, desde a gerência das Farmácias Hospitalares públicas, privadas e dos hospitais de campanha direcionados para o enfrentamento pandêmico, ofertando cuidados, orientações e educação em saúde que englobam a atenção farmacêutica. Logo, o objetivo da pesquisa foi analisar como vem sendo a Assistência Farmacêutica no âmbito hospitalar, na linha de frente no combate a COVID-19, no município de GurupiTO. Trata-se de um estudo quali-quantitativo, na qual foi aplicado um questionário eletrônico, respondido pelos profissionais farmacêuticos hospitalares de ambos os sexos que atuam na linha de frente do COVID-19, no município de Gurupi-TO. Foram selecionados 27 farmacêuticos, destes $80,9 \%$ atuavam na linha de frente no combate a COVID-19. Entre as dificuldades enfrentadas, $57,1 \%$ destacaram o estoque de medicações não condizente com o número de pacientes. Em relação a assistência farmacêutica hospitalar prestada em pacientes internados com COVID-19 no Hospital Regional de Gurupi, ocorre através da dispensação de medicamentos receitados pelos médicos, sendo feita a orientação ao paciente. Conclui-se que a atuação do Farmacêutico necessita de atenção, para a sua efetiva participação, pois é importante a sua colaboração na eficácia da recuperação da saúde dos clientes dentro e fora do ambiente hospitalar.

Palavras-chave: Infecções por Coronavírus. Assistência Farmacêutica. Hidroxicloroquina. Cloroquina. Eritromicina.

\section{ABSTRACT}

Hospital pharmacists play a fundamental role in periods of epidemic crisis, from the management of public and private Hospital Pharmacies and campaign hospitals aimed at fighting the pandemic, offering health care, guidance and education that encompass pharmaceutical care. Therefore, the objective of the research was to analyze how Pharmaceutical Assistance has been in the hospital environment, in the front line in the fight against COVID-19, in the municipality of Gurupi-TO. This is a qualitative e quantitative study, in which an electronic questionnaire was applied, answered by hospital pharmacists of both genders who work in the front line of COVID-19, in the city of Gurupi-TO. Twenty-seven pharmacists were selected, of which $80.9 \%$ worked on the front line in the fight against COVID-19. Among the difficulties faced, $57.1 \%$ highlighted the stock of medications not consistent with the number of patients. Regarding hospital pharmaceutical care provided to patients hospitalized with COVID-19 at the Regional Hospital of Gurupi, it occurs through the dispensing of medicines prescribed by doctors, with patient orientation being provided. It is concluded that the role of pharmacists needs attention for their effective participation, as their collaboration in the effective recovery of the health of clients inside and outside the hospital environment is important.

Keywords: Coronavirus Infections. Pharmaceutical Services. Hydroxychloroquine. Chloroquine. Azithromycin.
${ }^{1}$ Acadêmica do curso de farmácia na Universidade de GurupiUNIRG.

E-mail:

pamcristina06@hotmail.com

ORCID:https://orcid.org/00000003-4894-6818

${ }^{2}$ Acadêmica do curso de farmácia na Universidade de GurupiUNIRG.

E-mail: laisbarreira99@gmail.com

ORCID: https://orcid.org/00000002-6247-0265

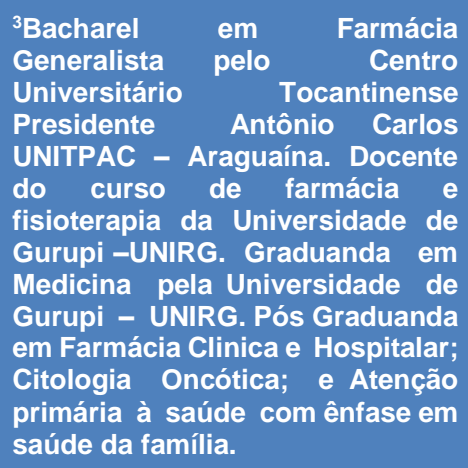

E-mail:

iessykavviana@gmail.com

ORCID:https://orcid.org/0000$\underline{0003-2842-0878}$

${ }^{4}$ Bacharel em enfermagem pela Universidade de Gurupi-UNIRG. Especialista em Ginecologia e Obstetrícia (FAVENI). Docente do Centro Universitário UNIPLAN.

E-mail:

profa.hallineiurema@gmail.com

ORCID: https://orcid.org/00000001-9865-3285 
DOI 10.18606/2318-1419/amazonia.sci.health.v9n4p17-28 Revista Amazônia Science \& Health
REIS, PCC. SILVA, LB. FRANCO, JVV. JUREMA, HC.

Análise da Assistência Farmacêutica Hospitalar na linha de frente no combate a COVID-19.

\section{INTRODUCÃO}

No final do ano de 2019, em uma das províncias centrais da China, surgiram alguns casos de uma doença, que até então, foi definida como um tipo de Pneumonia grave de origem imprecisa. O que levou os cientistas locais a realizarem pesquisas emergenciais, a fim de descobrirem maiores informações a respeito dessa nova patologia. Tempos depois, após muitos esforços foi verificado que se tratava de um vírus, que foi denominado SARSCoV-2. ${ }^{1}$

O Coronavírus, trata-se de uma família virótica composta por diferentes tipos de cargas virais, é responsável por provocar um quadro clínico que pode se manifestar-se em quadros assintomáticos e até mesmo em complicações respiratórias graves. ${ }^{2} \mathrm{O}$ nome SarsCoV-2, trata-se de é um vírus da Família Cononaviridae, com material genético composto por RNA de fita simples positivo, envolvido por cápsula de lipoproteína, contendo proteína Spike ou proteína-S fortemente ligada à enzima Enzima de Conversão de Angiotensina, Tipo 2 (ACE-2), que possui predileção pelas células pulmonares humanas. ${ }^{3}$

A transmissão da COVID-19, ocorre através do contato de um indivíduo contaminado, com um indivíduo susceptível, por meio do contato deste com gotículas e/ou aerossóis emanados através da tosse ou espirro. Logo, pela facilidade de transmissão do vírus, a OMS, deliberou sobre as principais medidas de prevenção e controle da doença, entre elas, o uso de máscaras, Equipamentos de Proteção Individual (EPI), higienização das mãos, distanciamento social e estado de quarentena por meios de decretos. ${ }^{1}$

Para a identificação e diagnóstico dos casos de COVID-19, foram adotados Exames de Biologia Molecular que constatam a presença do Ácido Ribonucleico (RNA) do vírus, no caso em específico, por meio do teste RT-PCR. A coleta do material biológico é feita mediante as secreções respiratórias, encontradas na região da nasofaringe, que é coletado utilizando um swab combinado ou pela amostra de uma secreção respiratória. ${ }^{4}$

Embora, no decorrer do ano de 2020, tenham surgido várias especulações sobre métodos terapêuticos para amenizar os sinais e sintomas da doença, ainda não existe uma confirmação científica, voltada para os fármacos que podem prevenir ou sanar a COVID19. No entanto, há muitos estudos em desenvolvimento para analisar a potencialidade de diversos medicamentos e terapias já em uso para o tratamento de outras patologias (tais como, fármacos antimaláricos, antivirais, antiinflamatórios; e tratamentos alternativos); 
DOI 10.18606/2318-1419/amazonia.sci.health.v9n4p17-28 Revista Amazônia Science \& Health
REIS, PCC. SILVA, LB. FRANCO, JVV. JUREMA, HC.

Análise da Assistência Farmacêutica Hospitalar na linha de frente no combate a COVID-19.

além de estudos clínicos voltados a descoberta de novos remédios voltados para o combate ao SARS-CoV-2. ${ }^{5}$

Dentro do contexto abordado, os farmacêuticos hospitalares apresentam um papel fundamental neste período de crise epidêmica, possuindo uma considerável relevância desde a gerência das Farmácias Hospitalares públicas, privadas e dos hospitais de campanha direcionados para o enfrentamento ao COVID-19, ofertando cuidados, orientações e educação em saúde que englobam a atenção farmacêutica. ${ }^{1}$

O profissional Farmacêutico tem o compromisso de orientar sobre o uso racional de medicamentos, visto que, devido ao medo, falta de informações ou pelas famosas Fake News, muitos indivíduos estão se automedicando desenfreadamente por acreditarem erroneamente que através do uso de certas medicações, como a Cloroquina e Hidroxicloroquina, estarão se precavendo de contrair ou desenvolver complicações severas da COVID-19. ${ }^{1}$

Diante do cenário, onde o número de internação cresce diariamente, o Farmacêutico hospitalar, é imprescindível para a aplicação de estratégias sistematizadas, com foco na educação em saúde, mostrando de forma clara qual a indicação de determinado medicamento, pois a seleção de medicamentos é de importante sendo o eixo norteador para o planejamento e orientação das etapas seguintes deste ciclo e da ação clínica, evitando assim o comprometimento das funções fisiológicas dos indivíduos, que pode vir a ser outro grave problema de saúde pública. ${ }^{6}$

Este estudo justifica-se pelo interesse em demonstrar a importância e a atuação e assistência do profissional Farmacêutico, no ambiente hospitalar no enfrentamento à COVID-19. A partir da realização desta pesquisa, pretende-se também colaborar para a disseminação de informações que venham a contribuir para o combate ao Coronavírus. Além disso, é importante que a população veja o quanto é essencial o papel de um Farmacêutico Hospitalar, pois este tem atuado na linha de frente, durante a pandemia.

Com o avanço dos números de casos confirmados pelo Coronavírus e dentre eles um aumento exponencial de pacientes internados pela COVID-19, o profissional Farmacêutico Hospitalar juntamente com os outros profissionais que compõe a equipe multidisciplinar, devem atuar buscando a prevenção de agravos e complicações de saúde, a melhoria do quadro evolutivo bem como a cura do paciente. Logo, o objetivo principal é analisar como vem sendo a Assistência Farmacêutica no âmbito hospitalar, na linha de frente no combate a COVID-19. 
DOI 10.18606/2318-1419/amazonia.sci.health.v9n4p17-28
REIS, PCC. SILVA, LB. FRANCO, JVV. JUREMA, HC.

Análise da Assistência Farmacêutica Hospitalar na linha de frente no combate a COVID-19.

\section{MATERIAIS E METODOS}

O estudo trata-se de uma pesquisa quali-quantitativo, pois centra-se na objetividade e recorre a linguagem matemática para descrever as causas de um fenômeno, as relações entre variáveis, entre outras. Quanto à natureza, a pesquisa é aplicada, pois ao final do trabalho de campo e de posse dos resultados, evidenciou-se como vem sendo a Assistência Farmacêutica no âmbito hospitalar, frente aos casos moderados e graves de pacientes com COVID-19.

Quanto aos objetivos, trata-se de uma pesquisa descritiva, por meio dela, o pesquisador busca informações e pretende descrever os fatos e fenômenos de uma determinada realidade. ${ }^{7}$

Quanto aos procedimentos, é uma pesquisa de campo, na qual foi aplicado um questionário eletrônico, respondido pelos profissionais farmacêuticos hospitalares de ambos os sexos que atuam na linha de frente do COVID-19 no município de Gurupi-TO, sendo realizado por meio de um corte transversal, pois sua característica principal foi obter dados fidedignos e que permitiram alcançar resultados confiáveis, gerar novas hipóteses, e a partir de então, surgir novas investigações. ${ }^{8}$

A pesquisa teve início após a autorização em pose do número do processo de aprovação emitido pelo Comitê de Ética em Pesquisa (CEP) da Universidade de Gurupi UnirG no 4.941.362.

Foram incluídos na pesquisa profissionais farmacêuticos de ambos os sexos que atuam na linha de frente ao combate da COVID-19 e prestam serviços na área hospitalar, que aceitaram participar da pesquisa através do link do questionário eletrônico que foi encaminhado via Google Forms, além disso, foi utilizado como fonte secundária o encaminhamento do link do formulário de pesquisa, através dos grupos das respectivas turmas, no aplicativo de conversa (WhatsApp) ou e-mail, juntamente foi enviado o link do Termo do Consentimento Livre e Esclarecido (TCLE) para aceite no estudo.

Foram excluídos da pesquisa as Unidades Básicas de Saúde (UBS), Unidade de Pronto Atendimento (UPA) e Ambulatórios, por estes prestarem assistência mais voltada aos pacientes com sintomas leves da doença, bem como, os Farmacêuticos contratados e concursados que não aceitarem participar da pesquisa com a não assinatura do TCLE. 
DOI 10.18606/2318-1419/amazonia.sci.health.v9n4p17-28 Revista Amazônia Science \& Health
REIS, PCC. SILVA, LB. FRANCO, JVV. JUREMA, HC.

Análise da Assistência Farmacêutica Hospitalar na linha de frente no combate a COVID-19.

\section{RESULTADOS}

Segundo o Conselho Regional de Farmácia do Estado do Tocantins (CRF-TO), constam em seus registros um total de 2.057 (dois mil e cinquenta e sete) farmacêuticos inscritos ativos, dividido em provisórios, secundários e definitivos, sendo que com domicílio profissional em Gurupi são 207 (duzentos e sete) profissionais. ${ }^{9}$ Dessa forma, foram selecionados para esta pesquisa, apenas profissionais farmacêuticos inscritos no conselho referido anteriormente e que atuam na linha de frente do combate a COVID-19 na cidade de Gurupi-TO, lidando com os pacientes em estado leves, moderados e graves pela infecção do coronavírus. Uma amostragem por conveniência, pois consistiu em selecionar uma amostra da população que seja acessível, pela facilidade no acesso aos farmacêuticos de tal região.

Foram selecionados 27 (100\%) farmacêuticos no total que aceitaram a responder a pesquisa, sendo estes a maioria do sexo feminino 20 (74,1\%), e com especialidades 18 $(69,2 \%)$ onde a farmácia clínica apresentou-se em maior porcentagem $(57,9 \%)$ conforme o gráfico 1.

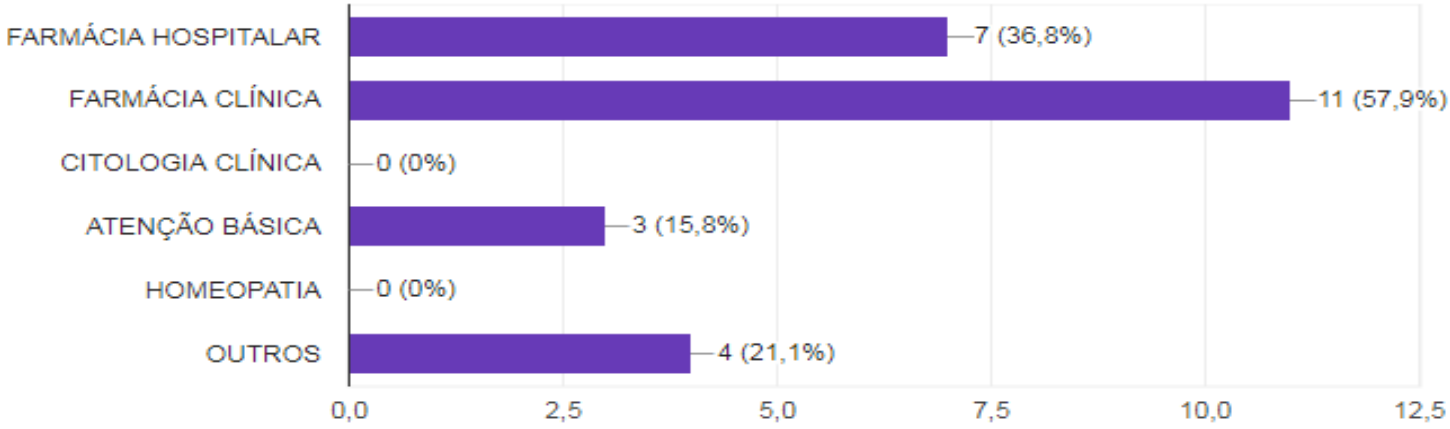

GRÁFICO 1: Especialidades dos Entrevistados.

Os farmacêuticos entrevistados, $(71,3 \%)$ prestavam serviços no município de Gurupi-TO. Sendo estes em diversos lugares, como UPA, Hospitais Particulares, Hospitais Regionais, UBS e Farmácias/Drogarias, todos atuando no tratamento da COVID-19. Conforme o gráfico 2, os dados levantados mostraram que a maioria presta serviços em Hospitais Regionais, bem como, em farmácias/drogarias no município de Gurupi-TO, com um percentil de (26,9\%), lidando com pacientes diretamente com COVID-19. 
DOI 10.18606/2318-1419/amazonia.sci.health.v9n4p17-28
REIS, PCC. SILVA, LB. FRANCO, JVV. JUREMA, HC.

Análise da Assistência Farmacêutica Hospitalar na linha de frente no combate a COVID-19.

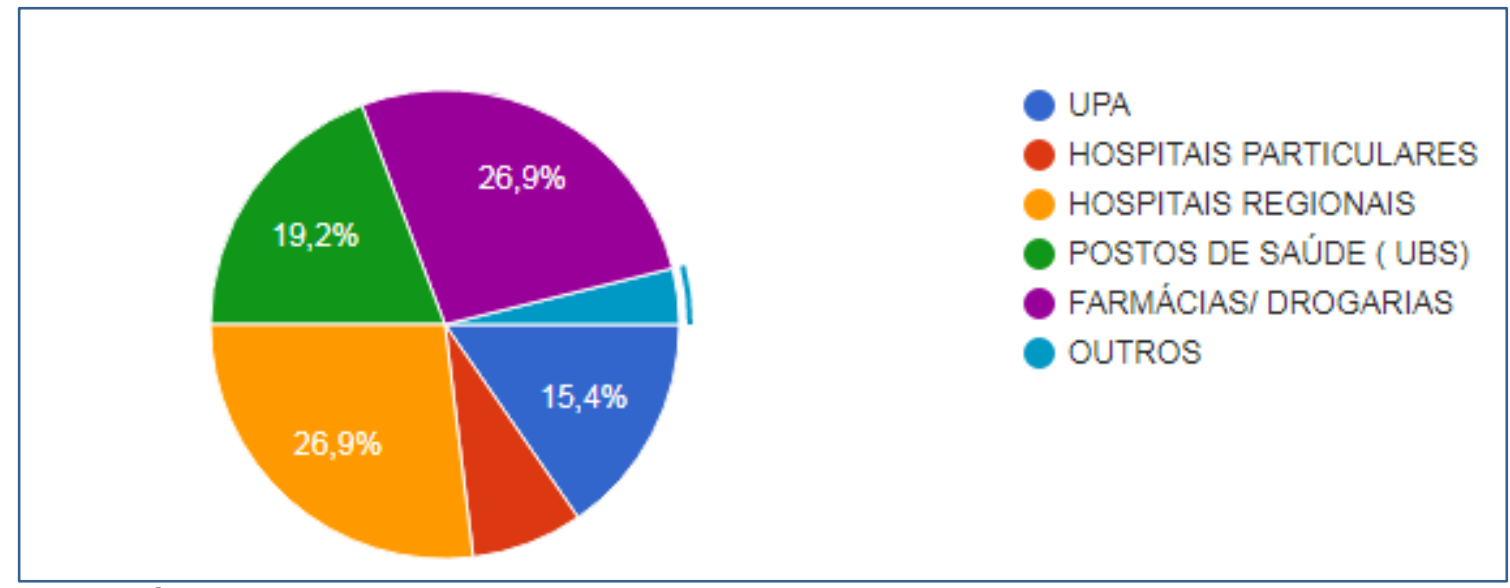

GRÁFICO 2: Local de trabalho/prestação de serviços dos farmacêuticos entrevistados.

A pesquisa mostrou que no total de farmacêuticos entrevistados, 80,9\% atuavam na linha de frente a COVID-19, na busca do tratamento para os pacientes que apresenta sintomas leves, moderados e graves.

Diante do gráfico 3, pacientes que são diagnosticados com COVID-19 apresentaram sintomas moderados sendo o mais frequente a febre com um percentual de $80 \%$, seguido da tosse seca $(76 \%)$, perda do paladar, cansaço e dor de cabeça com (72\%), perda de olfato (68\%) e ademais sintomas também foram relatados, tais como: diarreia, náuseas, oxigenação baixa e dor de garganta com percentuais acima de $20 \%$.

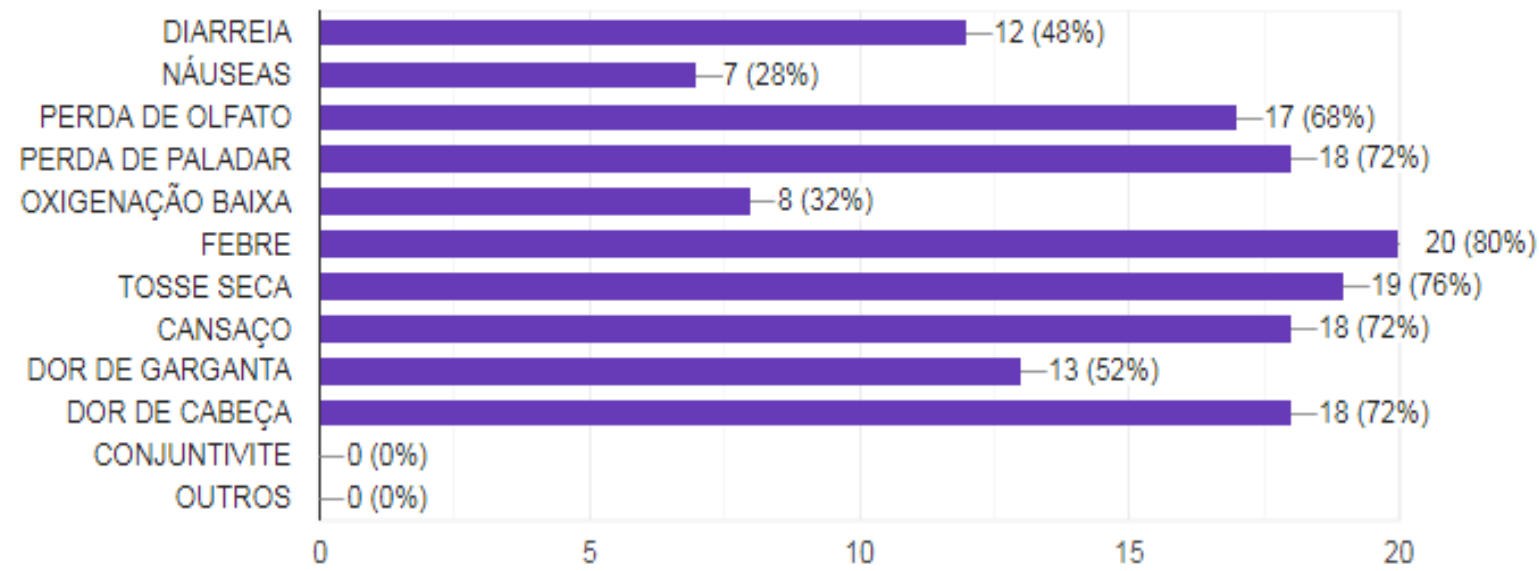

GRÁFICO 3: Sintomas moderados apresentados pelos pacientes com COVID-19.

Consta no gráfico 4 os sintomas graves pelos pacientes internados com coronavírus, sendo as mais comuns: dificuldade de respirar, dor ou pressão no peito perda de fala ou movimento confusão mental, alta temperatura, saturação baixa. 
DOI 10.18606/2318-1419/amazonia.sci.health.v9n4p17-28 Revista Amazônia Science \& Health 2021, Vol. 9, № 4
REIS, PCC. SILVA, LB. FRANCO, JVV. JUREMA, HC.

Análise da Assistência Farmacêutica Hospitalar na linha de frente no combate a COVID-19.

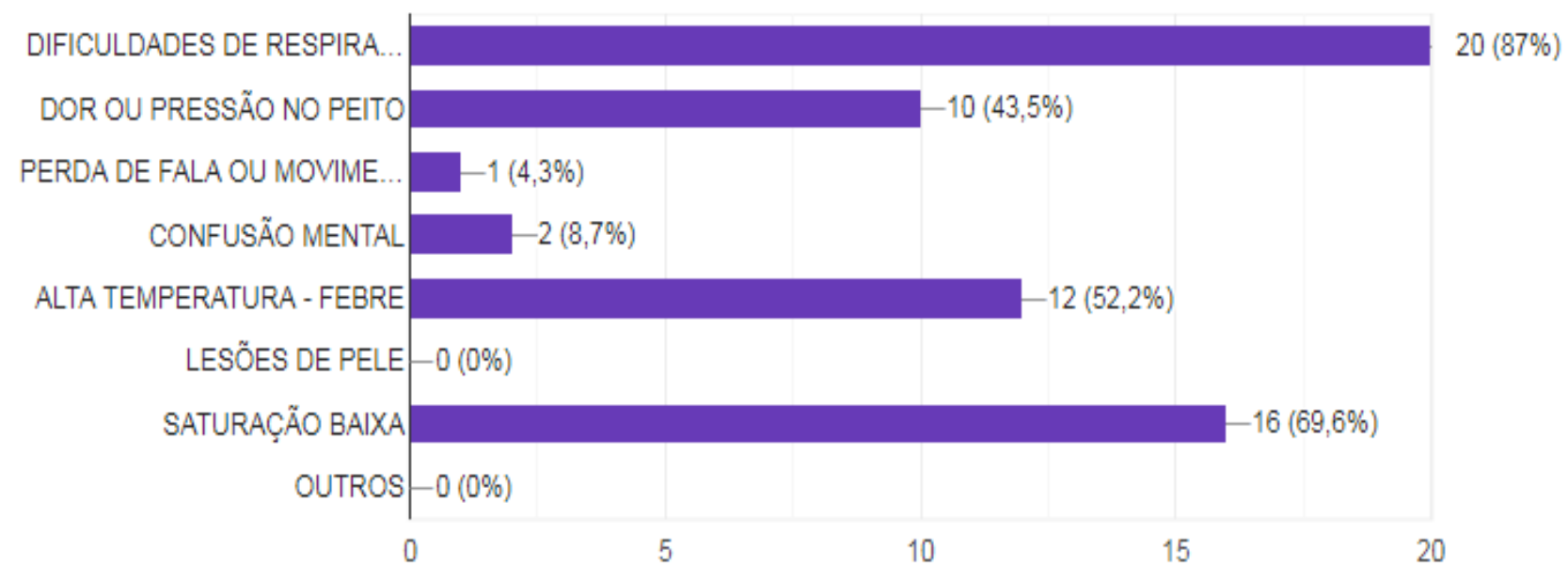

GRÁFICO 4: Sintomas graves apresentados pelos pacientes internados com COVID-19.

Diante disso, o principal objetivo das farmácias hospitalares é melhorar a qualidade da assistência prestada aos pacientes e promover o uso seguro e razoável de medicamentos e produtos afins. ${ }^{10}$ Dessa forma a pesquisa mostra que a assistência farmacêutica hospitalar prestada em pacientes internados com COVID-19 no Hospital Regional de Gurupi (HRG) acontece por meio da dispensação de medicamentos receitados pelos médicos, com orientação ao paciente, conforme o gráfico 5 .

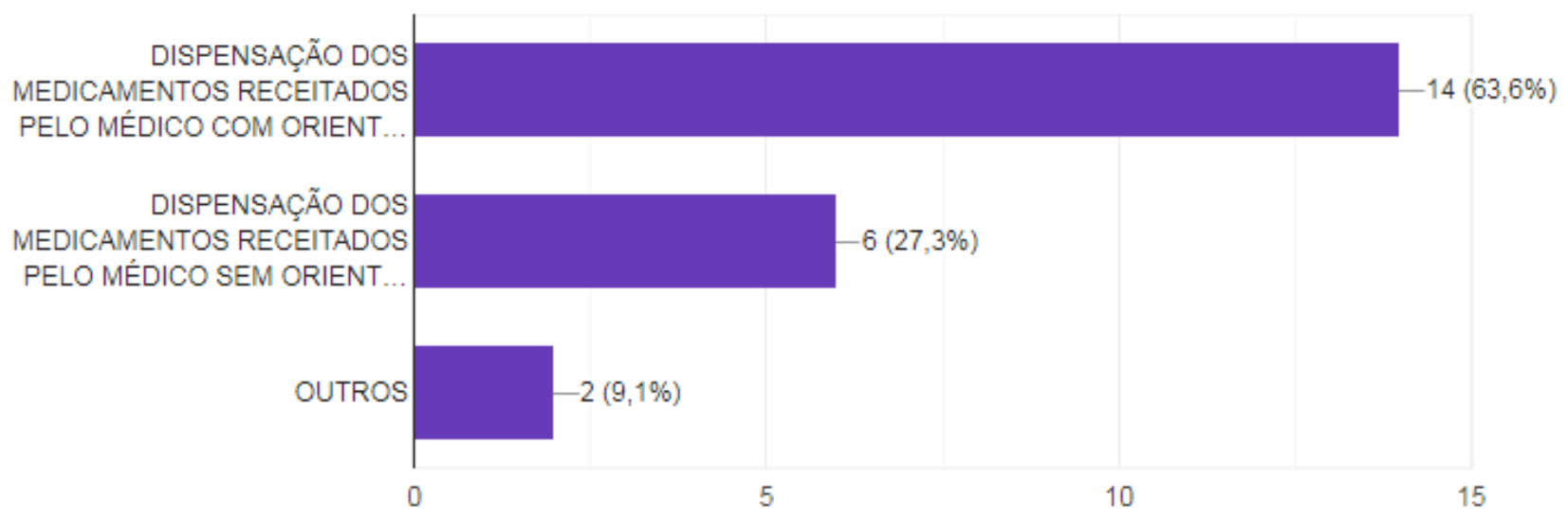

GRÁFICO 5: Assistência farmacêutica para os pacientes internados com COVID-19 no HRG.

No decorrer da pandemia pela COVID-19, as Farmácias Hospitalares tiveram que se reestruturarem e organizarem para realizarem o controle dos estoques, medicamentos e outros produtos para a saúde. Variáveis como armazenamento de medicamentos e tempo de estoque são fatores que provocaram diretamente o aumento do preço das farmácias hospitalares. A Associação Brasileira de Farmácia Hospitalar (ABFH), conceituou-o como 
DOI 10.18606/2318-1419/amazonia.sci.health.v9n4p17-28 Revista Amazônia Science \& Health 2021, Vol. 9, № 4
REIS, PCC. SILVA, LB. FRANCO, JVV. JUREMA, HC.

Análise da Assistência Farmacêutica Hospitalar na linha de frente no combate a COVID-19.

"um departamento clínico, administrativo e econômico dirigido por um profissional médico, hierarquicamente vinculado à gestão do hospital e funcionalmente integrado aos demais departamentos de atendimento ao paciente". ${ }^{11}$

Dessa maneira, os medicamentos ao chegarem no hospital, inicia-se a etapa de armazenamento que engloba desde o recebimento, a guarda, a estocagem respeitando as características físico-químicas do produto, o controle para que não ocorram danos ou roubos, avaliação do prazo de validade dos produtos, a entrega e a ordem de entrada e saída. $^{12}$

Assim a pesquisa confirma, para que haja uma boa qualidade no atendimento adequado e com êxito é necessária uma estrutura que parte da física a equipe multidisciplinar capacitada, pois conforme o gráfico 6, aponta as dificuldades enfrentadas pelo farmacêutico hospitalar diante da COVID-19, sendo o percentual maior $(57,1 \%)$ foi destacado o estoque não condizente com o número de pacientes.

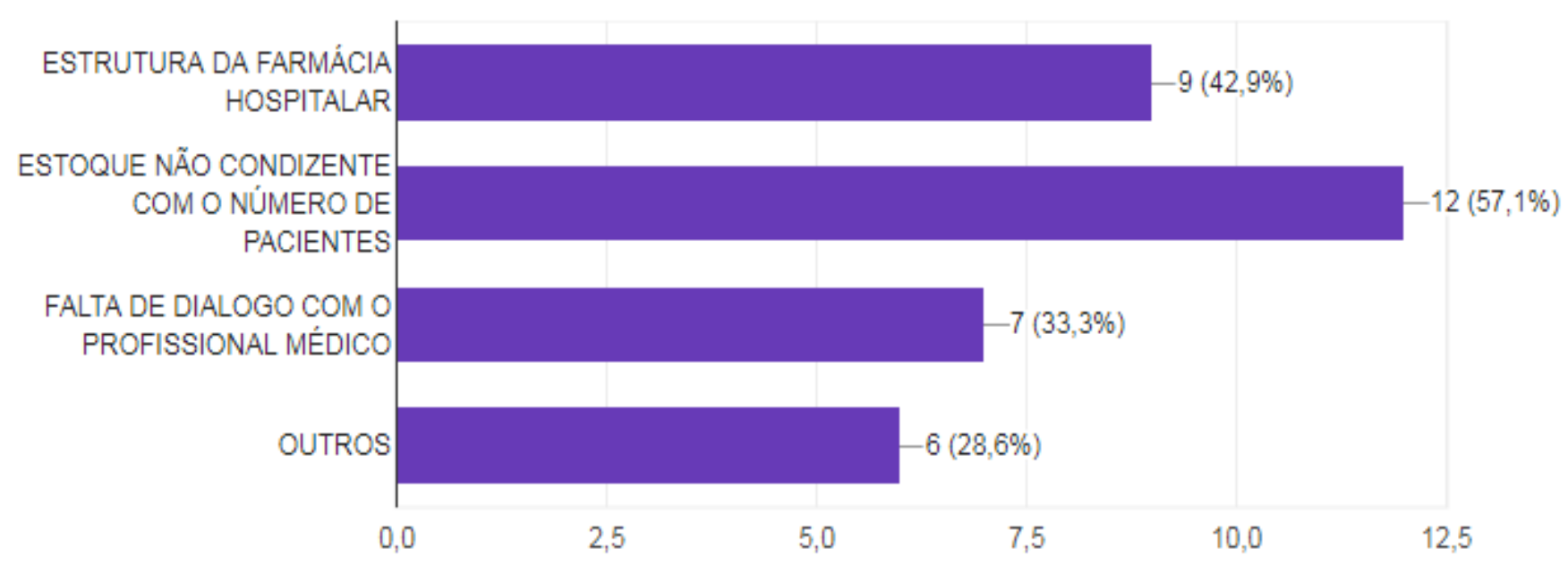

GRÁFICO 6: Dificuldades enfrentadas pelo farmacêutico hospitalar diante da COVID-19

\section{DISCUSSÃO}

Uma pneumonia de origem desconhecida teve seu primeiro caso detectado no final do ano de 2019 em uma cidade da província chinesa chamada Wuhan ${ }^{4}$. Por meio de análises epidemiológicas, verificou-se que a maioria dos pacientes alegaram ter visitado o Mercado de Frutos do Mar de Huanan, onde diversos animais silvestres eram comercializados. ${ }^{13}$

O primeiro caso no Brasil foi confirmado no dia 26 de fevereiro de 2020, em São Paulo, após um homem retornar de viagem da Itália e apresentar os sintomas 
DOI 10.18606/2318-1419/amazonia.sci.health.v9n4p17-28 Revista Amazônia Science \& Health
REIS, PCC. SILVA, LB. FRANCO, JVV. JUREMA, HC.

Análise da Assistência Farmacêutica Hospitalar na linha de frente no combate a COVID-19.

característicos do Coronavírus, sendo o caso confirmado após a realização de exames laboratoriais. ${ }^{14}$

Essa doença possui por natureza o comprometimento das funções respiratórias, possuindo manifestações clínicas iniciais comparadas a uma Síndrome Gripal (SG), entre elas podem estar presentes, a cefaleia, tontura, febre, tosse, mialgia ou fadiga, expectoração, dor ou inflamação da garganta, dispneia, diarreia e vômitos ou náuseas. Valendo ressaltar, que o vírus pode comprometer outros órgãos e/ou sistemas além dos pulmões, como por exemplo, o trato gastrointestinal, os rins, o fígado, e até mesmo o cérebro. De acordo com a literatura, boa parte dos casos são de pessoas que apresentam os quadros assintomáticos e leves da patologia. Sendo os casos mais graves, manifestados principalmente em idosos e pessoas com outros comprometimentos crônicos de saúde. ${ }^{15}$

O vírus possui uma alta facilidade de transmissão, tanto é que entre os meses de janeiro e fevereiro já haviam sido notificados novos casos em muitos países, de diferentes continentes, o que levou a Organização Mundial da Saúde (OMS), ainda no mês de fevereiro, declarar o estado de Emergência de Saúde Pública de Importância Internacional (ESPII). Porém, esse status logo foi atualizado, pois o vírus alastrou-se, sendo identificados novos casos diariamente de forma exponencial em novos países, levando a organização, a declarar a condição de pandemia, no início do mês de março de $2020{ }^{4}$

Medicamentos sujeitos a um controle especial, em destaque a hidroxicloroquina, devido ao seu consumo indiscriminado e conhecimento de seus efeitos adversos por meio da RDC n 351/2020, a mesma, passou a ser um tipo de controle diferente, em receitas comuns com validade de 30 dias, o medicamento conforme a Portaria SVS/MS no 344/1998, sendo assim armazenado em sala específica ou em armário exclusivo para medicamentos controlados trancado por chave ou similar sendo responsabilidade do farmacêutico e no ato da sua dispensa, a Notificação de Receita não será exigida para pacientes internados nos estabelecimentos hospitalares, porém a dispensação se fará somente com a receita ou outro documento equivalente (prescrição diária de medicamento), subscrita em papel privativo do estabelecimento. ${ }^{12}$

Na farmácia hospitalar a distribuição deve ocorrer através do Sistema de distribuição individualizado (dose individualizada), prática mais aplicável à Unidade de Terapia Intensiva (UTI), onde dentro de um período de 24 horas os medicamentos são distribuídos ao paciente, porém atualmente já se fala na organização deste processo em turnos (manhã, tarde e noite). O sistema individualizado de distribuição pode ser realizado pelo método 
DOI 10.18606/2318-1419/amazonia.sci.health.v9n4p17-28 Revista Amazônia Science \& Health
REIS, PCC. SILVA, LB. FRANCO, JVV. JUREMA, HC.

Análise da Assistência Farmacêutica Hospitalar na linha de frente no combate a COVID-19.

direto onde a cópia da prescrição médica chega à farmácia do hospital, sendo esta forma mais segura, ou pelo método indireto onde a equipe de enfermagem realiza a transcrição da receita e repassa a farmácia. ${ }^{12}$

De acordo com a OMS, o papel dos farmacêuticos no sistema de saúde inclui controle regulatório, incluindo farmácias comunitárias, farmácias hospitalares, indústria farmacêutica, atividades acadêmicas, treinamento de outros profissionais de saúde e gestão de medicamentos de pesquisa. Em todas essas áreas, o objetivo do farmacêutico é garantir a terapia medicamentosa ideal, facilitando a produção, o fornecimento e o controle de medicamentos e produtos para a saúde, além de fornecer informações e conselhos às pessoas que prescrevem ou usam medicamentos. ${ }^{11}$

A dispensação é um ato farmacêutico regulamentada pela Lei nํ⒌991/1973 que se faz mediante o fornecimento de medicamentos e outras especialidades farmacêuticas ao consumidor geralmente mediante apresentação de receita por profissional habilitado e orientação para promoção do uso racional de medicamentos. Visando uma melhora na qualidade de vida, a assistência farmacêutica também é responsável pelo cuidado farmacêutico e serviços clínicos assistenciais, assim o farmacêutico exerce sua atuação clínica promovendo maiores benefícios ao paciente e reduzindo os riscos relacionados a farmacoterapia. Dentre as atividades clínicas do farmacêutico que podem ser incorporadas ao processo destacamos a prevenção e resolução de problemas relacionados a medicamento (PRM) reduzindo o tempo de permanência do paciente no hospital e a morbimortalidade, reduzindo assim os custos hospitalares e os recursos advindos do setor público. ${ }^{12}$

Assim, algumas atribuições do farmacêutico sofrem atualizações durante um momento pandêmico, como o atual. A tarefa do farmacêutico é garantir que os medicamentos usados no hospital sejam usados corretamente, porque ainda não existe um medicamento aprovado pela SARS-CoV-2, que seja seguro e eficaz. Portanto, cabe ao profissional, monitorar quaisquer reações adversas que possam ocorrer nesses pacientes, principalmente, no caso de lesões hepáticas. ${ }^{1}$

Contudo, o Farmacêutico hospitalar deve atuar juntamente com os outros profissionais de saúde procurando, cada vez mais, melhorar a assistência prestada ao paciente. Dessa forma, devido o farmacêutico hospitalar fazer parte do conjunto de profissionais da saúde, o próprio colabora diretamente na terapia farmacológica e na dispensação correta de medicamentos. ${ }^{16}$ Acredita-se também que a Farmácia Hospitalar, 
DOI 10.18606/2318-1419/amazonia.sci.health.v9n4p17-28 Revista Amazônia Science \& Health 2021, Vol. 9, № 4
REIS, PCC. SILVA, LB. FRANCO, JVV. JUREMA, HC.

Análise da Assistência Farmacêutica Hospitalar na linha de frente no combate a COVID-19.

assim como os demais serviços pertencentes a unidade hospitalar, precisam adotar técnicas de segurança, tendo em vista a qualidade nos serviços prestados.

\section{CONSIDERAÇÕES FINAIS}

Respondendo ao objetivo que norteou a presente pesquisa, foi possível inferir que a Assistência Farmacêutica no âmbito hospitalar, na linha de frente no combate a COVID-19, baseou-se na dispensação de medicamentos receitados pelos médicos, e a realização de orientações ao paciente, por parte dos farmacêuticos, o que demonstrou uma certa limitação da atuação destes profissionais.

Entre as dificuldades enfrentadas pelos participantes do estudo diante da pandemia, estiveram a estrutura da farmácia hospitalar; o estoque não condizente com o número de pacientes; e a falta de diálogo entre o farmacêutico e médico, o que revela uma deficiência na prestação da assistência multiprofissional, que em momentos como o da crise sanitária, deveriam atuar de forma aproximada, buscando uma atenção qualificada aos clientes. Afinal, o profissional Farmacêutico Hospitalar juntamente com os outros profissionais que compõe a equipe, devem atuar buscando a prevenção de agravos e complicações de saúde, a melhoria do quadro evolutivo, bem como, a cura do paciente.

Sendo assim, observa-se que a atuação do Farmacêutico ainda necessita de uma maior atenção, para a sua efetiva participação, pois é importante a sua colaboração na eficácia da recuperação da saúde dos clientes dentro e fora do ambiente hospitalar, visto que, o próprio é quem deve realizar o acompanhamento para se evitar erros na administração de medicações, no suporte a elaboração de terapia medicamentosa, na dispensação dos fármacos e na educação farmacológica aos clientes, especialmente durante uma crise pandêmica, em que é relevante a assistência de todos os profissionais que compõe a equipe multidisciplinar.

\section{REFERÉNCIAS}

1.Silva LMC, Araújo JL. Atuação do farmacêutico clínico e comunitário frente a pandemia da COVID-19. Research, Society and Development. 2020; 9(7): 1-14.

2.Lima RSH, Silva CC. A importância do oficial farmacêutico no enfrentamento da pandemia de COVID-19 no âmbito do Exército Brasileiro. Biblioteca Digital do Exército. 2020; 1(1): 125. 
DOI 10.18606/2318-1419/amazonia.sci.health.v9n4p17-28 Revista Amazônia Science \& Health 2021, Vol. 9, № 4
REIS, PCC. SILVA, LB. FRANCO, JVV. JUREMA, HC.

Análise da Assistência Farmacêutica Hospitalar na linha de frente no combate a COVID-19.

3.Nogueira JVD. Conhecendo a Origem do Sars-Cov-2 (COVID-19). Revista Saúde e Meio Ambiente. 2020; 11(2): 115-124.

4.Franco JVV, Oliveira TF. O avanço do COVID-19 na Amazônia Legal: Uma análise do crescimento de casos na cidade de Gurupi-Tocantins. Amazônia: Science \& Health. 2020; 8(2): 64-76.

5.Duarte DB, Coelho LJ, Nascimento GNL. Atualidades da farmacoterapia da COVID-19. Desafios-Revista Interdisciplinar da Universidade Federal do Tocantins. 2020; 7(3): 81-89.

6.Tritany RF, Tritany ÉF. Serviços Farmacêuticos no Enfrentamento à COVID-19: Uma Revisão Integrativa da Literatura. Saúde em Redes. 2020; 6(2): 7-24.

7.Gerhardt TE, Silveira DT. Métodos de Pesquisa. Plageder, 1 ed., 2009.

8.Raimundo JZ, Echeimberg JO, Leone C. Research methodology topics: Cross-sectional studies. Journal of Human Growth and Development. 2018; 28(3): 356-360.

9.Conselho Regional de Farmácia do Tocantins (CRF-TO). Farmacêuticos inscritos ativos: provisórios, secundários e definitivos no Tocantins. 2020. [Acesso em 5 out. 2021]. Disponível em: < http://crfto.org.br/ >.

10.Reis WCT, Scopel CT, Correr CJ, Andrzeievski VMS. Análise das intervenções de farmacêuticos clínicos em um hospital de ensino terciário do Brasil. Einstein (São Paulo). 2013; 11(2): 190-196.

11.Rosa CDP, Berbare MHAO, Zucchi P. Inovação em saúde: atuação do farmacêutico hospitalar. In: II Simpósio Internacional de Gestão de Projetos e Simpósio Internacional de Inovação e Sustentabilidade. São Paulo-SP, 2014. p.1-12.

12.Monteiro FFC, Araújo IG, Santos RO, Nascimento RM, Loureiro LMA, Pessoa ETFP, Morais ACLN. Papel da assistência farmacêutica no tratamento de pacientes com COVID19 em hospital de referência no município de Fortaleza. Revista de Casos e Consultoria. 2020; 11(1): 1-21.

13.Cagnazzo TDO, Chiari-Andréo BG. Covid-19: Cuidados farmacêuticos durante a pandemia. Revista Brasileira Multidisciplinar. 2020; 23(1): 161-178.

14.Ministério da Saúde (Brasil). COVID-19 - Painel Coronavírus. Coronavírus Brasil. [Internet]. Brasília-DF, 2020a. [Acesso em: 20 mar. 2021]. Disponível em: <https://covid.saude.gov.br/>. Atualizado em 20 de mar. de 2021.

15.Monte LM, Mendes LA, Camargo RL, Gomes RSS, Silveira PHA, Seyfarth MSC, Silveira GRRA. Complicações atípicas e características clínico-epidemiológicas do COVID-19: uma revisão integrativa. Revista Eletrônica Acervo Saúde. 2020; 46(46): 1-12.

16.Freitas GRM, Luna-Leite MA, Castro MS, Heineck I. Principais dificuldades enfrentadas por farmacêuticos para exercerem suas atribuições clínicas no Brasil. Revista Brasileira de Farmácia Hospitalar e Serviços de Saúde. 2016; 7(3): 35-41 